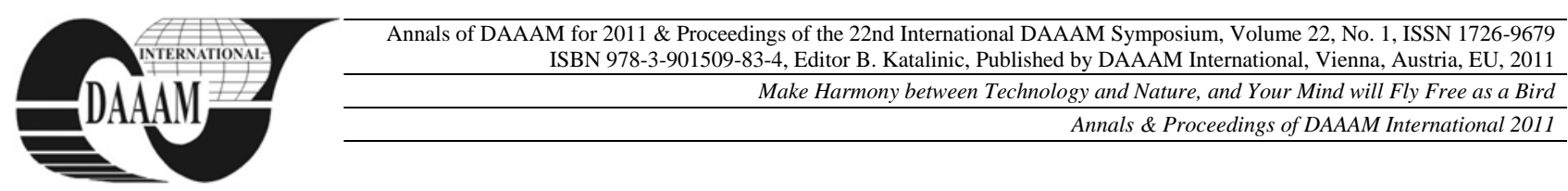

\title{
RIGID BODY ATTITUDE CONTROL WITH COMPLETE REJECTION OF UNKNOWN EXTERNAL DISTURBANCES
}

\author{
TOMIC, T[eodor]; KASAC, J[osip] \& MILIC, V[ladimir]
}

\begin{abstract}
In this paper a new class of rigid body attitude controllers is proposed. The proposed controllers provide asymptotic attitude stabilization with complete rejection of unknown external disturbances. The controller design is based on integral sliding-mode control strategy which, in contrast with standard sliding mode control approach, provides asymptotic tracking without chattering. Simulation results show favorable features of proposed controllers for stabilization of rigid body in presence of relatively large disturbances.

Key words: rigid body attitude control, disturbance rejection, integral sliding mode control, sliding PID control
\end{abstract}

\section{INTRODUCTION}

The rigid body attitude control problem (Wen \& KreutzDelgado, 1991) is relevant for a wide class of applications, e.g., helicopters, satellites, spacecrafts, and underwater vehicles maneuvering. The attitude stabilization problem in presence of external disturbances (Ding \& Li, 2009; Yamashita et al., 2004) has also attracted a great deal of interest, since the external disturbances are always of existence. Depending on particular applications, there are many unknown disturbances, such as gravitational torque, radiation torque, fluid turbulence and other environmental torques.

The continuous control laws, like robust $\mathrm{H}_{\infty}$ control and adaptive control cannot completely reject unknown external disturbances. On the other hand, the sliding-mode control approach (Kim at al., 1998) provides complete disturbance rejection. But, one of the drawbacks of sliding-mode control is the chattering problem which causes the high-frequency oscillations of control variables. For the rigid body control, chattering may excite high frequency modes and cause the structural failure. Chattering can be avoided by smoothing the control input by boundary layer method. But, such a continuous sliding-mode controller cannot achieve asymptotic stabilization since a steady state error is present.

In this paper we propose a control law for rigid body attitude stabilization in presence of large external disturbances which provides asymptotic stabilization without chattering. The proposed approach is based on concept of integral sliding mode or sliding PID control (Parra-Vega et al., 2003; Peng \& Chen, 2009). The main problem in direct application of standard sliding PID control strategy to rigid body attitude control is definition of position error in terms of Euler angles. In this paper we resolve this problem by defining attitude error in the form of vector products between actual and desired orientation, following similar argumentation as is described in (Milic et al., 2009).

\section{RIGID BODY ATTITUDE CONTROL}

We consider rotational motion of a single rigid body controlled by torque actuators, such as thrusters or propellers. The controller design is based on the assumption that the rigid body attitude and angular velocity are available for feedback and that the rigid body is controlled by three independent torque actuators.

\subsection{Rigid body attitude dynamics and kinematics}

The three-axis rotational dynamics of the rigid body are represented as

$$
\mathbf{J} \dot{\boldsymbol{\omega}}+\boldsymbol{\omega} \times(\mathbf{J} \boldsymbol{\omega})=\boldsymbol{\tau}+\mathbf{d},
$$

where $\mathbf{J}$ is the $3 \times 3$ inertia matrix, $\boldsymbol{\omega}$ is the $3 \times 1$ angular velocity vector, $\boldsymbol{\tau}$ is the $3 \times 1$ vector of actuator torques, and $\mathbf{d}$ is the $3 \times 1$ vector of unknown external disturbances.

The kinematics equations of the rigid body are given by

$$
\dot{\mathbf{x}}=\mathbf{\Omega}_{B} \boldsymbol{\omega},
$$

where $\mathbf{x}=\left[\begin{array}{lll}\phi & \theta & \psi\end{array}\right]^{\mathrm{T}}$ are Euler angles defined according to the $x y z$-convention, and

$$
\boldsymbol{\Omega}_{B}=\frac{1}{c_{2}}\left[\begin{array}{ccc}
c_{2} & s_{1} s_{2} & c_{1} s_{2} \\
0 & c_{1} c_{2} & -s_{1} c_{2} \\
0 & s_{1} & c_{1}
\end{array}\right],
$$

where $c_{i} \equiv \cos \left(x_{i}\right)$ and $s_{i} \equiv \sin \left(x_{i}\right), i=1,2,3$.

The objective of the control system is to bring the rigid body to the desired attitude $\mathbf{x}_{d}=\left[\phi_{d} \theta_{d} \psi_{d}\right]^{\mathrm{T}}$ starting from any initial condition, in presence of unknown external disturbances.

\subsection{Sliding PID controller}

The proposed control law has the following form

$$
\begin{gathered}
\boldsymbol{\tau}=\operatorname{sat}\left(\mathbf{K}_{P} \mathbf{s}+\mathbf{K}_{I} \mathbf{z} ; \boldsymbol{\tau}_{\max }\right), \\
\dot{\mathbf{z}}=\mathbf{s}+\mathbf{Q} \operatorname{sign}(\mathbf{s}),
\end{gathered}
$$

where sliding vector $\mathbf{s}$ is defined by

$$
\mathbf{S}=\alpha_{1} \mathbf{r}_{1} \times \mathbf{r}_{d 1}+\alpha_{2} \mathbf{r}_{2} \times \mathbf{r}_{d 2}-\boldsymbol{\omega},
$$

where

$$
\begin{aligned}
\mathbf{r}_{1}=\mathbf{R}_{y}(\theta) \mathbf{R}_{z}(\psi) \mathbf{e}_{1}, & \mathbf{r}_{d 1}=\mathbf{R}_{y}\left(\theta_{d}\right) \mathbf{R}_{z}\left(\psi_{d}\right) \mathbf{e}_{1}, \\
\mathbf{r}_{2}=\mathbf{R}_{x}(\phi) \mathbf{e}_{2}, & \mathbf{r}_{d 2}=\mathbf{R}_{x}\left(\phi_{d}\right) \mathbf{e}_{2}, \\
\mathbf{e}_{1}=\left[\begin{array}{lll}
1 & 0 & 0
\end{array}\right]^{T}, & \mathbf{e}_{2}=\left[\begin{array}{lll}
0 & 1 & 0
\end{array}\right]^{T},
\end{aligned}
$$

rotational matrices are

$$
\begin{gathered}
\mathbf{R}_{x}(\phi)=\left[\begin{array}{ccc}
1 & 0 & 0 \\
0 & c_{1} & s_{1} \\
0 & -s_{1} & c_{1}
\end{array}\right], \quad \mathbf{R}_{y}(\theta)=\left[\begin{array}{ccc}
c_{2} & 0 & s_{2} \\
0 & 1 & 0 \\
-s_{2} & 0 & c_{2}
\end{array}\right], \\
\mathbf{R}_{z}(\psi)=\left[\begin{array}{ccc}
c_{3} & s_{3} & 0 \\
-s_{3} & c_{3} & 0 \\
0 & 0 & 1
\end{array}\right],
\end{gathered}
$$

and saturation function is defined as

$$
\operatorname{sat}\left(x ; x_{\max }\right)=\left\{\begin{array}{ccc}
x_{\max } & \text { if } & x \geq x_{\max } \\
x & \text { if } & -x_{\max }<x<x_{\max } \\
-x_{\max } & \text { if } & x \leq-x_{\max }
\end{array}\right.
$$

The controller gains $\mathbf{K}_{P}, \mathbf{K}_{I}$, and $\mathbf{Q}$ are symmetric positivedefinite matrices, $\alpha_{1}$ and $\alpha_{2}$ are positive scalar parameters, and $\boldsymbol{\tau}_{\max }$ is saturation level of torque actuators. Note that in case of $\mathbf{Q}=\mathbf{0}$, sliding-mode PID controller becomes the standard (but nonlinear) PID controller. The controller behaviors is well- 
defined everywhere, except in singular positions defined by $\cos (\theta)=0$. The basic structure of integral PID controller is similar as in (Peng \& Chen, 2009) with main differences in the form of sliding variables. The sliding variable contains two nonlinear proportional terms in the form of vector products between actual and desired attitude, following similar argumentation as is described in (Milic et al., 2009). Such a proportional term induces the control torque which moves the rigid body in direction which decreases the angle between actual and desired orientation. Also, saturation function is included to prevent control signals with magnitudes larger then saturation level of actuators.
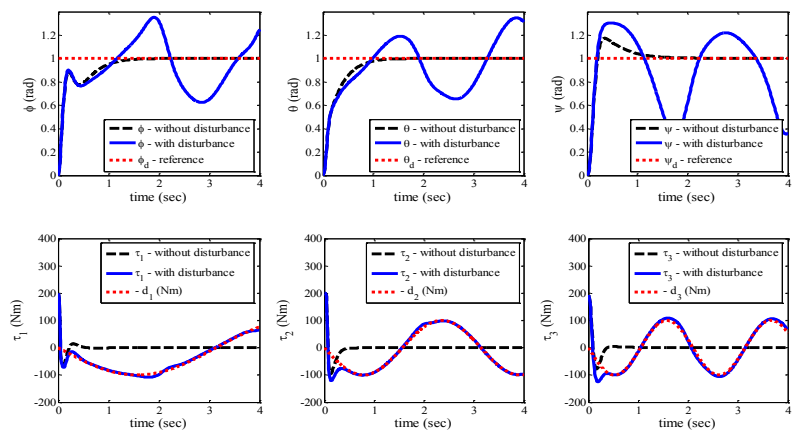

Fig. 1. Attitude and moments of rigid body in the closed-loop with standard PID controller $(\mathbf{Q}=\mathbf{0})$ in the case of external periodic disturbances

\section{SIMULATION RESULTS}

In this section the results of simulation verification of proposed control strategy to rigid body attitude stabilization are presented. The rigid body inertia matrix is $\mathrm{J}=\operatorname{diag}\{1,2,3\}$, desired attitude is $\mathbf{x}_{d}=\left[\begin{array}{lll}1 & 1 & 1\end{array}\right]^{\mathrm{T}}$, maximum value of actuator torques is $\tau_{\max }=200 \mathrm{Nm}$, and disturbance torque is $\mathbf{d}=$ $100[\sin (t) \sin (2 t) \sin (3 t)]^{\mathrm{T}} \mathrm{Nm}$. The controller parameters are: $\mathbf{K}_{P}=\operatorname{diag}\{100,100,100\}, \mathbf{K}_{I}=\operatorname{diag}\{100,100,100\}, \alpha_{1}=\alpha_{2}=$ $10, \mathbf{Q}=\operatorname{diag}\{10,10,10\}$.

In Fig. 1. we can see simulation results for standard PID controller $(\mathbf{Q}=\mathbf{0})$ in the case with and without large external periodic disturbances (with amplitude which is $50 \%$ of maximum available control torque). First, we can see that in the case without time-varying disturbances PID controller asymptotically stabilize rigid body attitude. Similar result can be obtained in the case of constant disturbances. But, in the case of time-varying disturbances the standard PID controller cannot stabilize the rigid body attitude. Moreover, the large disturbance cause large oscillations of rigid body attitude around desired stationary state.

In Fig. 2. we can see simulation results for sliding PID controller $(\mathbf{Q}=\operatorname{diag}\{10,10,10\})$ in the case with and without of external periodic disturbances. We can see that similar response is obtained for the case with and without of disturbances. In other words, sliding PID controller provide asymptotic stabilization of rigid body attitude completely rejecting external disturbances. The time-dependence of control torques (bottom subfigures in Fig. 2) shows that control torques exactly compensate the external disturbances, so that $\boldsymbol{\tau}+\mathbf{d}=\mathbf{0}$. On this way, the proposed controller can be used also as disturbance observer.

Simulation results for other choices of initial and final conditions (except singular positions defined by $\cos (\theta)=0$ ) show similar behavior. Also, controller shows high robustness to changes in system parameters.
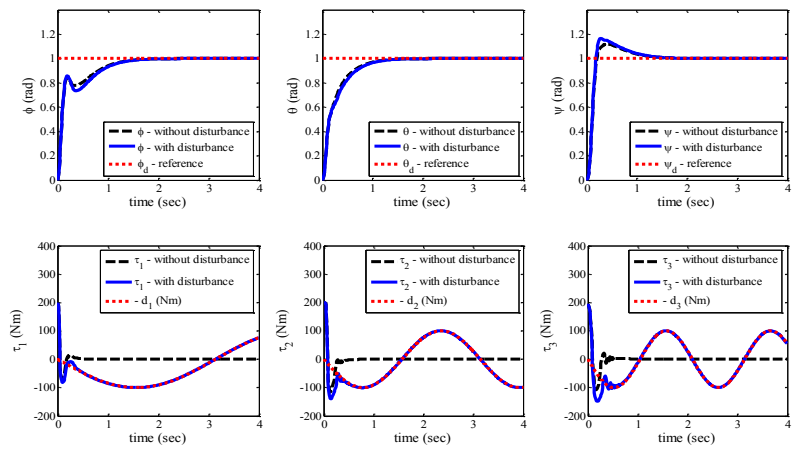

Fig. 2. Attitude and moments of rigid body in the closed-loop with sliding-mode PID controller $(\mathbf{Q}=\operatorname{diag}\{10,10,10\})$ in the case of external periodic disturbances

\section{CONCLUSION}

In this paper a control strategy for asymptotic stabilization of rigid body attitude based on integral sliding-mode approach is presented. The proposed controller provides total disturbance rejection without control variables chattering. The main problem of proposed control strategy is singular point which is characteristics for Euler representation of rigid body kinematics. Since the representation of rigid body kinematics using quaternions avoids singularity problems, the future work will be oriented toward extension of proposed control strategy using quaternion representation. Also, Lyapunov-based stability analysis will be applied with aim to provide exact controller tuning rules.

\section{REFERENCES}

Ding, S. \& Li, S. (2009). Stabilization of the attitude of a rigid spacecraft with external disturbances using finite-time control techniques, Aerospace Science and Technology, Vol. 13, pp. 256-265, ISSN 1270-9638.

Fossen, T. I. (1994). Guidance and Control of Ocean Vehicles, John Wiley \& Sons, ISBN 0-471-94113-1, New York

Kim, J.; Kim, J. \& Crassidis, J. L. (1998). Disturbance accommodating sliding mode controller for spacecraft attitude maneuvers, Advances in the Astronautical Sciences, Vol. 100, No. 1, pp. 141-153, ISSN 0065-3438

Milic, V.; Kasac, J. \& Situm, Z. (2009). Positioning a MultiRobot System Formation using Potential Field Method, Annals of DAAAM for 2009 \& Proceedings of the 20th International DAAAM Symposium, 25-28th November 2009, Vienna, Austria, ISSN 1726-9679, ISBN 978-3901509-70-4, Katalinic, B. (Ed.), pp. 1599-1600, Published by DAAAM International Vienna, Vienna

Parra-Vega, V.; Arimoto, S.; Liu, Y. H.; Hirzinger, G. \& Akella, P. (2003). Dynamic Sliding PID Control for Tracking of Robot Manipulators: Theory and Experiments, IEEE Transaction on Robotics and Automation, Vol. 19, pp. 967-976, ISSN 1042-296X

Peng, C-C. \& Chen, C-L. (2009). Dynamic controller design for a class of nonlinear uncertain systems subjected to timevarying disturbance, Nonlinear Dynamics, Vol. 57, No. 3, pp. 411-423, ISSN 0924-090X

Wen, J.T. \& Kreutz-Delgado, K. (1991). The Attitude Control Problem, IEEE Transaction on Automatic Control, Vol. 36, No. 10, pp. 1148-1162, ISSN 0018-9286

Yamashita, T.; Ogura, N.; Kurii, T. \& Hashimoto T. (2004). Improved satellite attitude control using a disturbance compensator, Acta Astronautica, Vol. 55, pp. 15-25, ISSN 0094-5765 